\title{
Quantitative techniques for imaging cells and tissues
}

\author{
Christopher S. von Bartheld • Fred S. Wouters
}

Published online: 14 March 2015

(C) Springer-Verlag Berlin Heidelberg 2015

\section{Introduction}

This special issue focuses on two major aspects of cell biology: imaging and quantitation. The history of cell biology has been tightly interwoven with imaging and in particular with advances in microscopy throughout the past centuries (Wolpert 1995; Evanko et al. 2009; Milestones Light Microscopy 2009). The invention of microscopes was instrumental in defining cell theory, centuries ago, and the boundaries of visualization advanced further with electron microscopy, decades ago. Applications of fluorescent confocal microscopy have enabled new ways of observation at the cellular, subcellular and even molecular level (still evolving to the current time; Waters and Wittmann 2014). Less appreciated is the importance of quantitative approaches in cell biology. Quantitation adds an important, often essential, new dimension to the scientific process/endeavor, as expressed in the famous quote by Lord Kelvin:

"In physical science a first essential step in the direction of learning any subject is to find principles of numerical reckoning and methods for practicably measuring some quality connected with it ... when you can measure what you are speaking about, and express it in numbers, you know something about it; but when you cannot

C. S. von Bartheld $(\bowtie)$

Department of Physiology and Cell Biology, Center of Biomedical

Research Excellence in Cell Biology, University of Nevada

School of Medicine, Mailstop 352, Reno, NV 89557, USA

e-mail: cvonbartheld@medicine.nevada.edu

\section{F. S. Wouters}

Department of Neuropathology, University Medical School, Göttingen University, and Center for Nanoscale Microscopy and Molecular Physiology of the Brain, Göttingen, Germany express it in numbers, your knowledge is of a meagre and unsatisfactory kind; it may be the beginning of knowledge, but you have scarcely, in your thoughts, advanced to the stage of science" (Thomson 1889).

Cell biology benefits in multiple ways from the inclusion of appropriate quantitative approaches. This is well established in some areas of cell biology, such as the quantification of cells or synapses in neuroscience (Haug 1986; Coggeshall and Lekan 1996; Evans et al. 2004), but less so in other fields of cell biology, such as immunocytochemistry, superresolution microscopy, and nanotechnology. The merging of cell biology and quantitative biology is considered an area in need of greater attention and further development (Liberali and Pelkmans 2012; Wall 2012; Shekhar et al. 2014).

Technical advances in cell biology usually happen first at the qualitative level (which structures and molecules can now be identified or localized at higher resolution?), which is followed by various delays with the exploration and development of quantitative methods to compare different states (how many molecules or particles are present? By how much are they increased or decreased in different conditions? How can the readout be quantified?). The development, testing and refinement of quantitative aspects of novel technologies can take significant lead time, and development of proper applications of quantitative technologies has followed different time courses in different fields of cell biology.

\section{Recognition of novel technical advances in cell biology by recent Nobel prizes}

New technologies create new frontiers. Advances have been remarkable in the past two decades in areas such as confocal 
microscopy, green fluorescent protein, nanotechnology (quantum dots), and super-resolution microscopy. This is reflected by a series of Nobel prizes that were awarded for new technological milestones: structure of macromolecules (in 2002), magnetic resonance imaging (in 2003), green fluorescent protein (in 2008), and, most recently, super-resolution microscopy (in 2014).

The recent surge in new developments in microscopy has opened new avenues of research with intriguing possibilities. A critical "mass" has been reached, in which this research drive poses new demands to advance in the directions just now enabled. The end of this scientific tidal wave is not yet in sight. Amidst these exciting new techniques, we present a special issue that addresses analytical, technical and chemical developments that accompany and/or lie at the basis of the new microscopy applications. Many perceived barriers (spatial resolution, speed, field size, detection limits, probe brightness) have fallen. Never before have we been able to see the microscopic world in which cells and tissues perform their tasks in so much detail, nor have we been able to extract so much information from biological specimens. Qualitative imaging can only provide snapshots of events and states without providing the tools for interpretation. Quantitation is key to our advances as it brings the necessary objectivity to understand rules and causal relationships between observable events.

We congratulate the 2014 Nobel laureates in chemistry, Eric Betzig, Stefan Hell and William Moerner, for their achievements in moving beyond the diffraction barrier, allowing light-based imaging on length scales that were, previously, only accessible via electron microscopy, but now in multiple colors, in 3D and in live specimens. Super-resolution techniques come in multiple flavors. Those that are based on localizing single emitters even provide information about their number. All these techniques, because of their dramatically reduced sampling volume, reduce the averaging of events in focus, providing a clearer view of distributions of behaviors. They are, therefore, also great tools for quantitative imaging. Our special issue contains three contributions that directly deal with super-resolution techniques, their possibilities and demands.

\section{Overview of the contributions to the special issue}

For this special issue of Cell and Tissue Research, we have asked experts in the fields of imaging, image analysis, and quantitation of cells and tissues to provide an update on the status of quantitative methods. These methods range from older methods, introduced decades ago, such as autoradiography and immunocytochemistry, to the most recent, such as super-resolution microscopy. Tried and tested quantitative techniques that were established long ago can and will be reinterpreted and adapted to meet the new measurement possibilities offered by the latest technologies. Heterogeneity in refinement of the quantitative methods is reflected in the various contributions of this issue of Cell and Tissue Research. For example, as elucidated in the contributions of Geuna and Herrera-Rincon (2015), Kubínová and Janáček (2015), and Mayhew (2015), proper counting of particles in sectioned tissues has been worked out over several decades, culminating with the design of the tools of stereology (Haug 1986; Coggeshall and Lekan 1996; Evans et al. 2004), but it took nearly a century and considerable effort by a large number of investigators to perfect the design-based approaches after the first cells were counted under the light microscope in the late 1800s (Blinkov and Glezer 1968; Haug 1986). Currently, the key stereological method papers enjoy exceptionally high citation rates, although the actual usage still lags behind, as revealed in the contribution by Geuna and Herrera-Rincon (2015).

Our special issue starts with the century-old problem of how to count particles in sectioned tissues. Geuna and Herrera-Rincon (2015) provide an update of the current status of the stereological tools, as applied to sections at the lightmicroscopic level, including an update of current usage of different counting techniques over the past decade. This is followed by the contribution of Kubínová and Janáček (2015) who examine the specific challenges and opportunities when applying stereological tools to confocal microscopy. The next review, by Herculano-Houzel et al. (2015), presents a novel alternative method of counting cells, the isotropic fractionator, which basically homogenizes tissue to a "soup", collects the nuclei, and samples and distinguishes neurons from non-neuronal cells by antibody-labeling. The advantages and disadvantages of this simple, yet elegant, approach are compared with stereology, including practical hands-on issues. We complete the "particle counting tool kit" with the contribution by Mayhew (2015). He explains how stereological methods are used for tissues at the ultrastructural level, again including several hands-on practical examples that guide the reader through each of the steps.

Staying with the important aspect of correlating lightmicroscopic and electron-microscopic levels of analysis, Giepmans and colleagues (Kuipers et al. 2015) present a novel genetic tool (FLIPPER) for labeling ("painting") and examination of molecules in cultured cells at the ultrastructural level that allows for optimal fixation and preservation of morphology, thus expanding the toolkit of researchers who wish to examine specific molecules and processes at the ultrastructural level. Such analyses can make use of nanotechnology, e.g., for tagging antibodies and other proteins. In this context, the current status and the emerging use of the quantification of nanoparticles (quantum dots) is covered by the contribution co-authored between the laboratories of Tania $\mathrm{Vu}$ and Diane Lidke (Vu et al. 2015). Nanotechnology is an emergent field 
that has a huge intersection with cell biology and promises fascinating progress not only in basic cell biology but also applied biology, including clinical areas such as diagnostics and therapeutics.

The decades-old field of autoradiography and in particular drug distribution is covered from a pharmaceutical/drug development perspective that reviews how autoradiographic approaches are essential to verify drug distribution and kinetics in animals and humans for the testing of new drugs (Solon 2015). Several decades after the introduction of immunocytochemistry, histopathologists are still in the process of defining and developing proper procedures for true quantification of immunolabels. With the current possibilities of visualizing single emitting fluorophores and achieving nano-scale localization, these questions are again becoming very acute. Likewise from a clinical perspective, we have an opportunity to hear the vision as laid out by a clinical pathologist, and specifically how immunocytochemistry can and must become more quantitative in the future (Taylor 2015). Digital data analysis and increased computing power now allows for much more efficient and advanced neuromorphometry, but, to optimize data documentation and interpretation in this new field, standardization is required, as explained in the "at a glance" article by Giorgio Ascoli and his colleagues (Parekh et al. 2015). This paper reviews how databases have become important tools in the dissemination and access to neuronal tracings of different cell types in different species.

Fluorescence detection is inherently more quantitative than the classical precipitation-based immunohistochemical stainings. Its linearity with concentration, its exceptional sensitivity with modern detectors - allowing single molecule detection which has been one of the pillars of the modern superresolution techniques - and its possibility for multiplexing multiple labels with different spectral separation characteristics in the same sample, puts fluorescence imaging at the foremost front of modern microscopy. A recent reinvention of a century-old idea for optical tomography, i.e. the selective imaging of a thin plane in the three-dimensional object, is called light sheet-based fluorescence microscopy (LSFM) (Pampaloni et al. 2015). By the orthogonal arrangement of illumination and emission paths, multiple advantages that are important for minimally invasive "deep imaging" of living specimens are gained: it provides optical sectioning, penetration depth is increased, illumination is restricted to the detection volume - which reduces probe destruction and phototoxicity. This method is now being used for a variety of applications, including "microtome-free histology."

The first paper on super-resolution techniques is by Bianchini et al. (2015) who provide an overview of the current state of the stimulated emission depletion (STED) technique. Here, the effective excitation volume is "squeezed down" from what can be achieved by lenses ("diffraction-limited") by overlaying the spot with an equally diffraction-limited beam that switches off fluorescence. The off beam is structured in the shape of a ring with zero illumination at its center. Pumping this beam closes the ring down, reducing the excitation spot down to the remaining zero-hole of the off beam. Progress has been made in resolution, achieving multi-color imaging and the required power of the off beam.

A second method to achieve super-resolution is discussed by Dedecker and colleagues (Vandenberg et al. 2015). In the so-called "localization methods", the fluorophores are made to glow sparsely enough to be imaged individually. A single emitter will not be able to be imaged as a point because, as in excitation, lenses cannot focus point images perfectly. However, the individual nature of the blurred spots allows the determination of their center, i.e. it is possible to point at the origin of the spot, which is the single emitter. Repetitive imaging of the sparsely fluorescing scene will thus deliver lists of coordinates for the single emitters, from which a synthetic image can be reconstructed. The methods for making fluorophores light up non-simultaneously, and the challenges in obtaining images from spots are discussed - this is inherently an issue of quantitation, and the possibilities are also considered in comparison to STED.

These applications demand unique properties from the fluorophores used. Higher resolution demands higher photon output. In STED, the fluorophore must be able to be switched off efficiently by appropriate illumination with the off beam. The fluorophore must be photostable at this wavelength, as high powers are needed to squeeze the off ring down. In localization microscopy, fluorophores must be switchable by light or must be made to "blink" at the appropriate interval to generate inhomogeneously sparse images of the single emitters. This requires chemical tricks. These properties are not always found in the fluorescent proteins. Bruchez and his colleague (Yan and Bruchez 2015) discuss the current state of the field of chemical labeling in biological tissues. Using chemical handles introduced to targets of interest in cells, chemical dyes can be introduced. These methods combine the precision of genetic targeting that the fluorescent proteins also possess, but with virtually limitless options for the actual fluorophore and its properties. These chemical approaches have been instrumental for the advance of superresolution microscopy of cells and can also be used to encode, in cells, quantitative assays for biochemical events.

The editors are confident that this collection of reviews covering the intersection of imaging, image analysis and quantitative approaches will be useful for cell biologists who wish to introduce or expand quantitative cell biology in their research and strive to better understand some of the new quantitative techniques related to imaging of cells and tissues - and delve into the real science as defined so befittingly by Lord Kelvin. 
Acknowledgments The authors thank the Editorial Assistant of Cell and Tissue Research, Ms. Maite Menés, for her excellent and effective coordination, as well as the Editor-in-Chief, Professor Klaus Unsicker, for his unwavering support. This project was made possible by grants from the National Institutes of Health: NS073113, NS079884, and GM103554 (CSvB); and from the Federal Ministry of Education and Research: 13 N9243 and 13 N10433 (FSW).

\section{References}

Bianchini P, Peres C, Oneto M, Galiani S, Vicidomini, Diaspro A (2015) STED nanoscopy: a glimpse into the future. Cell Tissue Res. doi:10. 1007/s00441-015-2146-3

Blinkov SM, Glezer II (1968) The human brain in figures and tables. A quantitative handbook. Plenum, New York

Coggeshall RE, Lekan HA (1996) Methods for determining numbers of cells and synapses: a case for more uniform standards of review. $\mathrm{J}$ Comp Neurol 364:6-15

Evanko D, Heinrichs A, Karlsson-Rosenthal C (2009) Editorial. Milestones in light microscopy. Nat Cell Biol 11:1165

Evans SM, Janson AM, Nyengaard JR (2004) Quantitative methods in neuroscience. Oxford University Press, New York

Geuna S, Herrera-Rincon C (2015) Update on stereology for light microscopy. Cell Tissue Res. doi:10.1007/s00441-015-2143-6

Haug H (1986) History of neuromorphometry. J Neurosci Methods 18:117

Herculano-Houzel S, von Bartheld CS, Miller DJ, Kaas JH (2015) How to count cells: the advantages and disadvantages of the isotropic fractionator compared with stereology. Cell Tissue Res. doi:10. 1007/s00441-015-2127-6

Kubínová L, Janáček J (2015) Confocal stereology - efficient tool for measurement of microscopic structures. Cell Tissue Res. doi:10. 1007/s00441-015-2138-3

Kuipers J, van Ham TJ, Kalicharan RD, Veenstra-Algra A, Sjollema KA, Dijk F, Schnell U, Giepmans BNG (2015) FLIPPER, a combinatorial probe for quantitative correlated live imaging and electron microscopy. Cell Tissue Res. doi:10.1007/s00441-015-2142-7
Liberali P, Pelkmans L (2012) Towards quantitative cell biology. Nat Cell Biol 14:1233

Mayhew TM (2015) Quantitative immunocytochemistry at the ultrastructural level: a stereology-based approach to molecular nanomorphomics. Cell Tissue Res. doi:10.1007/s00441-014-2038-y

Milestones Light Microscopy. 2009. Nat Cell Biol 11:S5-S22. (http:// www.nature.com/milestones/milelight/timeline.html) (accessed $1 / 15 / 2015)$

Pampaloni F, Chang BJ, Stelzer EHK (2015) Light sheet-based fluorescence microscopy (LSFM) for the quantitative imaging of cells and tissues. Cell Tissue Res. doi:10.1007/s00441-015-2144-5

Parekh R, Armañanzas R, Ascoli GA (2015) The importance of metadata to assess information content in digital reconstructions of neuronal morphology. Cell Tissue Res. doi:10.1007/s00441-014-2103-6

Shekhar S, Zhu L, Mazutis L, Sgro AE, Fai TG, Podolski M (2014) Quantitative biology: where modern biology meets physical sciences. Mol Biol Cell 25:3482-3485

Solon EG (2015) Autoradiography techniques and quantification of drug distribution. Cell Tissue Res. doi:10.1007/s00441-014-2093-4

Taylor CR (2015) Quantitative in situ proteomics; a proposed pathway for quantification of immunohistochemistry at the lightmicroscopic level. Cell Tissue Res. doi:10.1007/s00441-014-2089-0

Thomson W (1889) The practical applications of electricity. Electrical units of measurement. In: Nature Series. Popular Lectures and Addresses. (A lecture delivered at the Institution of Civil Engineers, May 3, 1883); Macmillan, London, 1:73-136

Vandenberg W, Leutenegger M, Lasser T, Hofkens J, Dedecker P (2015) Diffraction-unlimited imaging: from pretty pictures to hard numbers. Cell Tissue Res. doi:10.1007/s00441-014-2109-0

Vu TQ, Lam WY, Hatch EW, Lidke DS (2015) Quantum dots for quantitative imaging: from single molecules to tissue. Cell Tissue Res. doi:10.1007/s00441-014-2087-2

Wall ME (2012) Quantitative Biology: From Molecular to Cellular Systems. Chapman \& Hall CRC

Waters JC, Wittmann T (2014) Quantitative Imaging in Cell Biology, 1st edn. Methods in Cell Biology, vol 123. Academic, New York

Wolpert L (1995) Evolution of the cell theory. Philos Trans R Soc Lond B 349:227-233

Yan Q, Bruchez M (2015) Advances in chemical labeling of proteins in living cells. Cell Tissue Res. doi:10.1007/s00441-015-2145-4 